\title{
Damaged behavior under plane stress
}

\section{Numerical integration of elasto-viscoplastic-damage constitutive equations under plane stress condition}

\author{
Thomas Paris* — Khémaïs Saanouni** \\ * Commissariat à l'Énergie Atomique et aux Énergies Alternatives \\ Centre de Valduc \\ F-21120 Is-sur-Tille \\ thomas.paris@cea.fr \\ ** ICD/LASMIS, STMR UMR-CNRS 6279 \\ University of Technology of Troyes \\ 12, rue Marie Curie, BP2060 \\ F-10000 Troyes cedex \\ saanouni@utt.fr
}

\begin{abstract}
This paper deals with the numerical treatment of "advanced" elasto-viscoplasticdamage constitutive equations in the particular case of plane stress. The viscoplastic constitutive equations account for the mixed isotropic and kinematic non linear hardening and are fully coupled with the isotropic ductile damage. The viscous effect is indifferently described by a power function (Norton type) or an hyperbolic sine function. Different time integration schemes are used and compared to each other assuming plane stress condition, widely used when dealing with shell structures as well as to the $3 D$ reference case.

RÉSUMÉ. Cet article se propose d'aborder l'implémentation numérique d'une loi de comportement élastoviscoplastique couplée à l'endommagement dans le cas particulier de la contrainte plane. Les équations constitutives prennent en compte un écrouissage mixte à la fois isotrope et cinématique non linéaires couplés à l'endommagement ductile isotrope. Les effets de la viscosité peuvent être décrits soit par une fonction de type puissance (viscosité de Norton) soit par une fonction de type sinus hyperbolique. Différents algorithmes d'intégration des équations d'évolution sont comparés dans le cas des contraintes planes puis au cas de référence $3 D$ afin d'estimer l'erreur commise dans le cas des éléments coques pour la simulation de structures fines.

KEYWORDS: viscoplasticity, damage, plane stress, numerical integration.

MOTS-CLÉS : viscoplasticité, endommagement, contraintes planes, intégration numérique.
\end{abstract}

DOI:10.3166/EJCM.20.341-368 @ 2011 Lavoisier, Paris 


\section{Introduction}

Numerical integration of various types of plastic or viscoplastic constitutive equations has attracted many research works related to the development of the finite element analysis in 3D or 2D (plane stress, plane strain or axisymmetric cases). The reader is referred to the recent books (Simo et al., 1997; Doghri, 2000; Crisfield, 1991; Belytschko et al., 2000; Ibrahimbegovic, 2006) and references given there. Even if the 3D modeling of mechanical structures are required and easy to perform using the modern powerful computer facilities, the plane stress description is helpfully required when dealing with large mechanical components of thin sheets as in automotive structures using thin plates or thin shell elements.

Note that the plane strain and axisymmetric cases are well defined since they are particular case of the 3D numerical integration by reducing the stress components from six to only four. However, in the case of plane stress, these non-zero components are supplemented by the additional condition $\sigma_{33}=0$. For such case, it's well known that the use of backward Euler scheme is impossible or at least very difficult to perform, depending on the type of constitutive equations. Different treatments of the plane stress case can be done to enforce the constraint $\sigma_{33}=0$. This can be taken into account in the shape function of the element as proposed by De Borst (De Borst, 1991) or Besson (Besson et al., 1997). Another way is to consider this condition at the integration point level i.e using a special treatment of the constitutive equations. Different formulations can be used to achieve this goal which are reviewed in (Millard, 1995).

In the specialized literature, one can find three different ways to treat this problem. The first one imposes the plane stress constraint only during the plastic correction stage but not in the elastic prediction stage and leads to an additional equation with $\epsilon_{33}$ as additional unknown (Hammi, 2000; Lestriez, 2003) (among many others). The second one uses the same procedure but applied to both elastic prediction and plastic correction stages as formulated, for example, by Doghri (Doghri et al., 1995; Sawyer et al., 2001; Aravas, 1987). The third one, due to Simo (Simo et al., 1997; Simo et al., 1988), connects the constrained stress and its deviator using appropriate mapping technique. This leads to only one equation which is ideally suited for applying the general return-mapping algorithm. This method has been successfully used by different authors with complex models and various simulation cases from the original one of Simo (Waltz et al., 1990; Lee et al., 1998; Lee et al., 2001; Montans, 2004; Kumar et al., 2005; Valoroso et al., 2009a; Valoroso et al., 2009b) among many others.

In this paper, the above three different formulations of the plane stress algorithms are used to integrate a complete set of an elasto-viscoplastic constitutive equations fully coupled with ductile damage under isothermal conditions (Saanouni et al., 2003; Lestriez et al., 2004; Hammi, 2000; Lestriez, 2003). The three plane stress algorithms are applied to some simple examples (tension tests) and the results are compared with 3D calculations. To conclude, advantages and drawbacks of the different schemes are discussed and confronted on the lights of the fully coupled constitutive equations used here. 


\section{Numerical integration of the fully coupled elasto-viscoplastic damage constitutive equations}

\subsection{Summary of the coupled constitutive equation}

The used elasto-viscoplastic damage model presented here is based on the thermodynamical framework, using various state variables to describe phenomena such as isotropic and kinematic hardening, viscous and damage effects (Saanouni et al., 2003). In the isothermal case, the state variables are :

$$
\begin{aligned}
& \left(\varepsilon^{v p}, \boldsymbol{\sigma}\right) \quad \text { : viscoplastic strain and stress tensors } \\
& (\underset{\sim}{\boldsymbol{\alpha}}, \underset{\sim}{\mathbf{X}}) \quad \text { : kinematic hardening strain and stress tensors } \\
& (r, R) \quad \text { : isotropic hardening strain and stress } \\
& (D, Y) \quad \text { : isotropic damage and its associated force }
\end{aligned}
$$

The use of such a framework, allows to describe a fully coupled damage model really well established thanks to the formulation of state and dissipation potentials. Dealing with objective finite strain formulation, an updated Lagrangian description for which the total small strain rate tensor $\underset{\sim}{\dot{\varepsilon}}$ is transformed to its Eulerian counterpart $\underset{\sim}{\mathrm{D}}$, defined as the symmetric part of the Eulerian velocity gradient tensor, is followed (Dogui, 1989; Aravas et al., 1991).

\subsubsection{State equations}

The state equations, defining the force-like state variables with isotropic damage effect are given by (Saanouni et al., 1994; Saanouni et al., 2003) :

$$
\begin{aligned}
& \underset{\sim}{\sigma}=(1-D) \underset{\sim}{\boldsymbol{\Lambda}}:{\underset{\sim}{\varepsilon}}^{e} \\
& \underset{\sim}{\mathbf{X}}=(1-D) \frac{2}{3} C \underset{\sim}{\boldsymbol{\alpha}} \\
& R=(1-D) Q r \\
& Y=\frac{1}{2} \varepsilon^{e}: \underset{\sim}{\boldsymbol{\Lambda}}: \boldsymbol{\sim}^{e}+\frac{1}{3} C \underset{\sim}{\boldsymbol{\alpha}}: \underset{\sim}{\boldsymbol{\alpha}}+\frac{1}{2} Q r^{2}
\end{aligned}
$$

where $\underset{\sim}{\mathbf{\Lambda}}=2 \mu \underset{\sim}{\mathbf{1}}+\lambda \underset{\sim}{\mathbf{1}} \otimes \underset{\sim}{\mathbf{1}}$ is the elastic stiffness fourth order tensor with $\lambda$ and $\mu$ are Lame's elasticity coefficients, $\mathrm{Q}$ and $\mathrm{C}$ are the isotropic and kinematic hardening modulus respectively. 


\subsubsection{Evolution equations}

In the framework of the non associative theory (Lemaitre et al., 1985), the evolution equations related to the selected dissipative phenomena including the damage effect, are summarized here after (Saanouni et al., 1994; Saanouni et al., 2003) :

$$
\begin{aligned}
& \underset{\sim}{\mathbf{D}}=\dot{\sim}^{e}+{\underset{\sim}{\mathbf{D}}}^{v p} \\
& {\underset{\sim}{\mathbf{D}}}^{v p}=\frac{\dot{\lambda}_{v p}}{\sqrt{1-D}} \underset{\sim}{\mathbf{n}} \quad \text { with } \underset{\sim}{\mathbf{n}}=\frac{3}{2} \frac{\underset{\sim}{\mathbf{S}}-\underset{\sim}{\mathbf{X}}}{\|\underset{\sim}{\boldsymbol{\sigma}}-\underset{\sim}{\mathbf{X}}\|} \\
& \underset{\sim}{\boldsymbol{\alpha}}=\dot{\lambda}_{v p}\left(\frac{\mathbf{\sim}}{\sqrt{1-D}}-a \underset{\sim}{\boldsymbol{\mathbf { \alpha }}}\right) \\
& \dot{r}=\dot{\lambda}_{v p}\left(\frac{1}{\sqrt{1-D}}-b r\right) \\
& \dot{D}=\frac{\dot{\lambda}_{v p}}{(1-D)^{\beta+\frac{1}{2}}}\left\langle\frac{Y-Y_{0}}{S}\right\rangle^{s}
\end{aligned}
$$

where $\dot{\varepsilon}^{e}$ is the Jaumann (objective) derivative of the elastic small strain tensor and $\mathbf{D}^{v p}$ is the rotated Eulerian finite viscoplastic strain rate tensor. $\mathrm{a}, \mathrm{b}$ are the non linearity coefficients of kinematic and isotropic hardening respectively, $\beta, \mathrm{S}, \mathrm{s}, Y_{0}$ are material parameters characterizing the ductile damage evolution.

In this equations, $\dot{\lambda}_{v p}$ is the viscoplastic "multiplier" depending on the viscosity type :

- for the Norton viscosity function

$$
\dot{\lambda}_{v p}=\left\langle\frac{f}{K}\right\rangle^{n}
$$

- for the hyperbolic sine viscosity function

$$
\dot{\lambda}_{v p}=K_{1} \sinh \left\langle\frac{f}{K_{2}}\right\rangle
$$

Consequently, the viscous stress is given by :

- for the Norton viscosity function

$$
\sigma_{v}=K\left(\dot{\lambda}_{v p}\right)^{\frac{1}{n}}
$$

- for the hyperbolic sine viscosity function

$$
\sigma_{v}=K_{2} \operatorname{arcsinh}\left(\frac{\dot{\lambda}_{v p}}{K_{1}}\right)
$$

Many other viscosity functions can be used and different formulations are compared in (Chaboche, 2007). 
All these tensor variables are rotated using an appropriate rotation tensor in order to fulfill the objectivity requirement (Dogui, 1989; Badreddine, 2006).

Note that in this time dependent plasticity, the Ordinary Differential Equations (ODE) [6] to [9] are valid if and only if the following viscoplastic yield condition is fulfilled (Paris, 2008) :

$$
f_{v}=\|\underset{\sim}{\boldsymbol{\sigma}}-\underset{\sim}{\mathbf{X}}\|-R-\sqrt{1-D}\left(\sigma_{y}+\sigma_{v}\right)=0
$$

without any additional constraint (i.e no Kuhn-Tucker condition as in time independent plasticity).

\subsection{Stress computation scheme in $3 D$}

When discretized by the displacement based (Galerkin type) finite element method, the equilibrium equations lead to a highly non linear algebraic system (Belytschko et al., 2000). In this work, this non linear system is solved thanks to the explicit dynamic scheme more suited for problems containing damage induced softening in order to avoid the use of tangent stiffness matrix (Saanouni et al., 2003). However, to calculate the internal forces vector, the computation of the stress tensor, and consequently all state variables, is required at the end of each time increment.

If the classical incremental time discretization procedure is used, the overall loading path applied during the time interval $\left[t_{0} ; t_{f}\right]$ is decomposed into several non constant time steps $\left[t_{n} ; t_{n+1}=t_{n}+\Delta t\right]$.

The four ODE to be numerically integrated at each quadrature point of each finite element are Equations [6] to [9], in such manner that Equation [14] is fulfilled. To do that, the classical elastic prediction viscoplastic correction scheme will be used.

First of all, we recall that the above four ODE can be classified in two types. The Equations [6] and [9] have the form :

$$
\begin{aligned}
\forall t \in\left[t_{n} ; t_{n+1}\right] \\
\qquad\left\{\begin{array}{l}
\underset{\sim}{\mathbf{y}}=\varphi(\underset{\sim}{\mathbf{y}}, t) \\
\underset{\sim}{\mathbf{y}}\left(t_{n}\right)=\underset{\sim}{\mathbf{y}_{n}}
\end{array}\right.
\end{aligned}
$$

and the Equations [7] and [8] have the form of the so called Euler differential equation :

$$
\begin{aligned}
\forall t \in\left[t_{n} ; t_{n+1}\right] \\
\left\{\begin{array}{l}
\underset{\sim}{\mathbf{y}}=\varphi(\underset{\sim}{\mathbf{y}}, t)\left(\underset{\sim}{\phi}\left(t_{\sim}^{\mathbf{y}}, t\right)-\underset{\sim}{\mathbf{y}}\right) \\
\underset{\sim}{\mathbf{y}}\left(t_{n}\right)=\underset{\sim}{\mathbf{y}_{n}}
\end{array}\right.
\end{aligned}
$$


By using the classical $\theta$-method, the solution of [15] is :

$$
\underset{\sim}{\mathbf{y}_{n+\theta}}={\underset{\sim}{\mathbf{y}}}_{n}+\Delta t\left(\theta \dot{\sim}_{n+1}^{\dot{\mathbf{y}}_{n+1}}+(1-\theta) \underset{\sim n}{\dot{\mathbf{y}}_{n}}\right)
$$

while the exact solution of Equation [16] has the following form (Walker et al., 1991) :

$$
\underset{\sim}{\mathbf{y}_{n+\theta}}={\underset{\sim}{\mathbf{y}} n}_{n} e^{-\theta \varphi\left(\mathbf{y}_{n+\theta}\right) \Delta t}+\left(1-e^{-\theta \varphi\left(\mathbf{y}_{n+\theta}\right) \Delta t}\right) \underset{\sim}{\phi} \underset{\sim}{\left(\mathbf{y}_{n+\theta}\right)}
$$

Now applying Equation [17] to Equations [6] and [9], and Equation [18] to Equations [7] and [8] in the case of fully implicit backward Euler scheme defined by $\theta=1$ allows to write the overall constitutive equations at $t_{n+1}$ in the following form :

$$
\begin{aligned}
& {\underset{\sim}{\boldsymbol{\sigma}}}_{n+1}=\left(1-D_{n+1}\right) \underset{\sim}{\boldsymbol{\Lambda}}:{\underset{\sim}{\boldsymbol{\varepsilon}^{e}+1}}_{n}^{e} \\
& \underset{\sim}{\boldsymbol{\alpha}_{n+1}}=\underset{\sim n}{\underset{\boldsymbol{\alpha}}{n}} e^{-a \Delta \lambda_{v p}}+\frac{\underset{\sim}{\mathbf{n}_{n+1}}}{a \sqrt{1-D_{n+1}}}\left(1-e^{-a \Delta \lambda_{v p}}\right) \\
& r_{n+1}=r_{n} e^{-b \Delta \lambda_{v p}}+\frac{1}{b \sqrt{1-D_{n+1}}}\left(1-e^{-b \Delta \lambda_{v p}}\right) \\
& {\underset{\sim}{\varepsilon}}_{n+1}^{v p}={\underset{\sim}{\varepsilon}}_{n}^{v p}+\Delta \lambda_{v p} \frac{\stackrel{\mathbf{n}}{\sim}+1_{n+1}}{\sqrt{1-D_{n+1}}} \\
& D_{n+1}=D_{n}+\frac{\Delta \lambda_{v p}}{\left(1-D_{n+1}\right)^{\beta+\frac{1}{2}}}\left\langle\frac{Y_{n+1}-Y_{0}}{S}\right\rangle^{s}
\end{aligned}
$$

All these quantities, used in conjunction with the state Equations [1] to [4], must fulfill the yield condition at the end of each time increment $t_{n+1}$ :

$$
f_{v_{n+1}}=\left\|\boldsymbol{\sigma}_{n+1}-\underset{\sim}{\mathbf{X}_{n+1}}\right\|-R_{n+1}-\sqrt{1-D_{n+1}}\left(\sigma_{y}+\sigma_{v_{n+1}}\right)=0 \text { [24] }
$$

By using the well known elastic prediction and viscoplastic correction scheme the closest point projection algorithm can be constructed (Ortiz et al., 1986; Simo et $a l ., 1997)$ allowing the computation of the overall state variables at $t_{n+1}$. However, it is easy to show that the problem is reduced to solve only two scalar equations with 
respect to two scalar unknowns namely, in this case, $\Delta \lambda_{v p}$ and $D_{n+1}$. Those two equations are :

$$
\begin{aligned}
\bar{f}_{v}= & \left\|{\underset{\sim}{n+1}}_{n}^{\star}-\mathbf{\sim}_{n} e^{-a \Delta \lambda_{v p}}\right\|-\frac{1-D_{n}}{\sqrt{1-D_{n+1}}}\left(3 \mu \Delta \lambda_{v p}\right. \\
& +\frac{\sqrt{1-D_{n+1}}}{1-D_{n}} R_{n} e^{-b \Delta \lambda_{v p}}+\frac{C}{a}\left(1-e^{-a \Delta \lambda_{v p}}\right) \\
& \left.+\frac{Q}{b}\left(1-e^{-b \Delta \lambda_{v p}}\right)+\sigma_{y}+\sigma_{v_{n+1}}\right)=0 \\
g_{D} & D_{n+1}-D_{n} \\
& -\frac{\Delta \lambda_{v p}}{\left(1-D_{n+1}\right)^{\beta+\frac{1}{2}}}\left\langle\frac{Y_{n+1}\left(D_{n+1}, \Delta \lambda_{v p}\right)-Y_{0}}{S}\right\rangle^{s} \\
= & 0
\end{aligned}
$$

The components denoted by $\left({ }^{\star}\right)$ refer to the elastic prediction related quantities. Following the idea of Lestriez and Saanouni (Lestriez, 2003), Equation [26] can be simplified and written under the form given by Equation [27]. This allows the calculation of $D_{n+1}$ functions of $D_{n}$ and $\Delta \lambda_{v p}$ at each iteration and injected into [25]. This hypothesis is very efficient to save CPU time and doesn't change the mechanical response up to the very end of the behavior (final fracture).

$$
\begin{aligned}
g_{D} & =D_{n+1}-D_{n}-\frac{\Delta \lambda_{v p}}{\left(1-D_{n}\right)^{\beta+\frac{1}{2}}}\left\langle\frac{Y_{n+1}\left(D_{n}, \Delta \lambda_{v p}\right)-Y_{0}}{S}\right\rangle^{s} \\
& =0
\end{aligned}
$$

Consequently, only one scalar non linear equation is solved by a classical iterative Newton-Raphson scheme to find the single unknown $\Delta \lambda_{v p}$ from Equation [25] since $D_{n+1}$ is computed from [27]. All the other quantities are easily deduced from the increment of the viscoplastic multiplier.

\subsection{Stress computation in plane stress case}

Now three different algorithms called CP1, CP2, CP3 are formulated in the particular cases where the plane stress assumption is valid. 


\subsubsection{Algorithm CP1}

Solving Equation [25] is enough in the 3D and plane strain cases. However, the plane stress case needs an additional equation to be solved. A new unknown, $\Delta \varepsilon_{33}$, is added and has to fulfill an additional constraint $\sigma_{i 3}=0$. The subscript i3 refers to the out of plane components in $2 \mathrm{D}$ case. The two equations system that has to be solved becomes,

$$
\begin{aligned}
\bar{f}_{v}\left(\Delta \lambda_{v p}, \Delta \varepsilon_{33}\right) & =\left\|\underset{\sim}{\mathbf{S}_{n+1}}-\underset{\sim}{\mathbf{X}} n e^{-a \Delta \lambda_{v p}}\right\| \\
& -\frac{1-D_{n}}{\sqrt{1-D_{n+1}}}\left(3 \mu \Delta \lambda_{v p}\right. \\
& +\frac{\sqrt{1-D_{n+1}}}{1-D_{n}} R_{n} e^{-b \Delta \lambda_{v p}} \\
& +\frac{C}{a}\left(1-e^{-a \Delta \lambda_{v p}}\right) \\
& \left.+\frac{Q}{b}\left(1-e^{-b \Delta \lambda_{v p}}\right)+\sigma_{y}+\sigma_{v_{n+1}}\right)=0 \\
h_{n+1}\left(\Delta \lambda_{v p}, \Delta \varepsilon_{33}\right) & =\Delta \varepsilon_{33_{n+1}}+\frac{\nu}{1-\nu}\left(\Delta \varepsilon_{11_{n+1}}+\Delta \varepsilon_{22_{n+1}}\right) \\
& -\frac{1-2 \nu}{1-\nu} \Delta \varepsilon_{33_{n+1}}^{v p}=0
\end{aligned}
$$

To solve the previous system (Equations [28] and [29]), a Newton-Raphson algorithm is used and needs to compute the gradient of the flow rule and the hardening rule with respect to $\Delta \varepsilon_{33}$ and $\Delta \lambda_{v p}$. The use of $\Delta \varepsilon_{33}$, during the elastic prediction stage, is shown to be very important to guarantee a stable response as pointed out in Section 2.3 .2 .

\subsubsection{Algorithm CP2}

As mentioned before, the best way to ensure a stable response in any case is to realize both an elastic prediction on $\Delta \lambda_{v p}(=0)$ and $\Delta \varepsilon_{33}\left(=\Delta \varepsilon_{33}^{\star}\right)$. Algorithm 1 summarizes the algorithm. For a detailed explanation, the reader is referred to the Appendix.

$$
\underset{\sim}{\mathbf{n}_{n+1}}=\frac{3}{2} \frac{{\underset{\sim}{n+1}}_{n}^{\star}-\underset{\sim}{\mathbf{X}} e^{-a \Delta \lambda_{v p}}+2 \mu\left(1-D_{n}\right)\left(\Delta \varepsilon_{33}-\Delta \varepsilon_{33}^{\star}\right){\underset{\sim}{\mathbf{1}}}^{D}}{\left\|{\underset{\sim}{\mathbf{S}}}_{n+1}^{\star}-\underset{\sim}{\mathbf{X}} e^{-a \Delta \lambda_{v p}}+2 \mu\left(1-D_{n}\right)\left(\Delta \varepsilon_{33}-\Delta \varepsilon_{33}^{\star}\right){\underset{\sim}{1}}^{D}\right\|}
$$




$$
\begin{aligned}
& \bar{f}_{v}\left(\Delta \lambda_{v p}, \Delta \varepsilon_{33}, D_{n+1}\right)= \\
& \left\|\left.\right|_{\sim} ^{\star} \mathbf{S}_{n+1}^{\star}-\underset{\sim}{\mathbf{X}} e^{-a \Delta \lambda_{v p}}+2 \mu\left(1-D_{n}\right)\left(\Delta \varepsilon_{33}-\Delta \varepsilon_{33}^{\star}\right) \underset{\sim 3}{\underset{1}{D}}\right\| \\
& -\left[R_{n} e^{-b \Delta \lambda_{v p}}+\frac{1-D_{n}}{\sqrt{1-D_{n+1}}}\left(\frac{Q}{b}\left(1-e^{-b \Delta \lambda_{v p}}\right)\right.\right. \\
& \left.\left.+\frac{C}{a}\left(1-e^{-a \Delta \lambda_{v p}}\right)+\sigma_{y}+\sigma_{v_{n+1}}+3 \mu \Delta \lambda_{v p}\right)\right]=0 \\
& h\left(\Delta \lambda_{v p}, \Delta \varepsilon_{33}, D_{n+1}, \mathbf{n}_{n+1}\right)=\frac{1}{1-D_{n}}\left[\left({\underset{\sim}{\mathbf{S}}}_{n+1}^{\star}\right)_{33}+\frac{1}{3} \sigma_{n+1}^{\star H}\right] \\
& -\frac{2 \mu \Delta \lambda_{v p}}{\sqrt{1-D_{n+1}}}\left({\underset{\sim}{n}}_{n+1}\right)_{33}\left(K+\frac{4}{3} \mu\right)\left(\Delta \varepsilon_{33}-\Delta \varepsilon_{33}^{\star}\right)=0
\end{aligned}
$$

where subscribe 33 refers to the out of plane component. The damage still explicitly computed from Equation [27].

REMARK. - If we impose $\Delta \varepsilon_{33}=\Delta \varepsilon_{33}^{\star}$ in Equations [30], [31] and [32], the equations system turn out to be the same as in $3 \mathrm{D}$ or plane strain cases.

This algorithmic treatment is really suited for the $3 \mathrm{D}$, plane strain and plane stress cases. As we can see (Equation [30]), additional terms appear in the normal to the yield surface with the plane stress assumption. This equation demonstrates, the well known fact, that the radial return is no longer available in this case (Dodds, 1987; Singh et al., 1999; Fuschi et al., 1992).

To solve this problem, in order to use the radial return, Simo suggested to connect the constrained plane stress subspace to the deviatoric one (Simo et al., 1997; Simo et al., 1986; Simo et al., 1988). In the next section, this basic idea is used to implement a specific algorithm dedicated to the model developed in this paper.

This algorithm illustrates the fact that if the out of plane deformation isn't taken into account in the prediction stage, the normal is wrong and the path followed to return to the yield surface isn't the good one.

\subsubsection{Algorithm CP3}

- Basic concept

As proposed first by Simo (Simo et al., 1986; Simo et al., 1997), a projection based algorithm can be used to treat the plane stress case. We will show that this treatment is also efficient to formulate an elasto-visco-plastic model with non linear isotropic and kinematic hardening fully coupled with damage with the plane stress 
Algorithm 1 Closest point projection algorithm in plane stress (CP2)

1. Initialize $\Delta \lambda_{v p}=0,{\underset{\sim}{n+1}}_{n p}^{v p}=\varepsilon_{n}^{v p}, \mathbf{\sim}_{n+1}=\mathbf{\sim}_{n}$,

$R_{n+1}=R_{n}, D_{n+1}=D_{n}, \Delta \varepsilon_{33}=\Delta \varepsilon_{33}^{\star}$

2. Compute the trial state

3. Check yield condition

$f_{n+1}^{\star}=\frac{\left\|\boldsymbol{\sigma}_{n+1}^{\star}-\mathbf{X}_{n}\right\|-R_{n}}{\sqrt{1-D_{n}}}-\sigma_{y}$

4. IF $f_{n+1}^{\star} \leq$ TOL THEN

Set $(.)_{n+1}^{\star}=(.)_{n+1}^{\star}$

\section{ELSE}

5. Iterate WHILE $\bar{f}_{v}^{(i)}\left(\Delta \lambda_{v p}^{(i)}, \Delta \varepsilon_{33}^{(i)}, D_{n+1}^{(i)}\right) \geq \mathrm{TOL}$ or $h^{(i)}\left(\Delta \lambda_{v p}^{(i)}, \Delta \varepsilon_{33}^{(i)}, D_{n+1}^{(i)}, \underset{\sim}{\mathbf{n}+1}(i) \geq \mathrm{TOL}\right.$

Calculate the gradient of the flow rule and hardening laws

$$
\delta \Delta \lambda_{v p}=\left(-\bar{f}_{v}^{(i)}-\frac{\partial \bar{f}_{v}^{(i)}}{\partial \Delta \varepsilon_{33}} \delta \Delta \varepsilon_{33}\right)\left(\frac{\partial \bar{f}_{v}^{(i)}}{\partial \Delta \lambda_{v p}}\right)^{-1}
$$

$\delta \Delta \varepsilon_{33}=$

$\left(h^{(i)} \frac{\partial \bar{f}_{v}}{\partial \Delta \lambda_{v p}}-\bar{f}_{v}^{(i)} \frac{\partial h^{(i)}}{\partial \Delta \lambda_{v p}}\right)\left(\frac{\partial \bar{f}_{v}^{(i)}}{\partial \Delta \varepsilon_{33}} \frac{\partial h^{(i)}}{\partial \Delta \lambda_{v p}}-\frac{\partial \bar{f}_{v}^{(i)}}{\partial \Delta \lambda_{v p}} \frac{\partial h^{(i)}}{\partial \Delta \varepsilon_{33}}\right)^{-1}$

$\Delta \lambda_{v p}^{(i+1)}=\Delta \lambda_{v p}^{(i)}+\delta \Delta \lambda_{v p}$

$\Delta \varepsilon_{33}^{(i+1)}=\Delta \varepsilon_{33}^{(i)}+\delta \Delta \varepsilon_{33}$

6. Update state variables and normal (Equation [30])

7.Set $\mathrm{i}=\mathrm{i}+1$ and GOTO 5 .

ENDIF

condition automatically enforced thanks to an appropriate mapping. This mapping $\underset{\sim}{\mathbf{P}}: S_{P} \rightarrow S_{D}$ allows to impose the consequence of plane stress condition over the 
deviatoric tensors. Here, $\underset{\sim}{\mathbf{P}}$ is a fourth order tensor, $S_{P}$ is the plane stress subspaces and $S_{D}$ is the deviatoric subspace respectively defined by :

$$
\begin{aligned}
& S_{P}=\left\{\underset{\sim}{\boldsymbol{\sigma}} \epsilon S \mid \sigma_{13}=\sigma_{23}=\sigma_{33}=0\right\} \\
& S_{D}=\left\{\underset{\sim}{\mathbf{S}} \epsilon S \mid S_{13}=S_{23}=0, \operatorname{Tr}[\underset{\sim}{\mathbf{S}}]=0\right\}
\end{aligned}
$$

If we write $\underset{\approx}{\mathbf{P}}$ and its inverse $\underset{\approx}{\mathbf{R}}$ in vector notation, we have :

$$
\underset{\sim}{\mathbf{P}}=\frac{1}{3}\left[\begin{array}{ccc}
2 & -1 & 0 \\
-1 & 2 & 0 \\
0 & 0 & 3
\end{array}\right], \quad \underset{\sim}{\mathbf{R}}=\left[\begin{array}{lll}
2 & 1 & 0 \\
1 & 2 & 0 \\
0 & 0 & 1
\end{array}\right]
$$

We can rephrase the state equations (Equations [1] to [4]) with the mapping consideration :

$$
\begin{aligned}
\underset{\sim}{\boldsymbol{\sigma}} & =(1-D) \underset{\sim}{\tilde{\Lambda}}:{\underset{\sim}{e}}^{e} \\
\underset{\sim}{\tilde{\mathbf{X}}} & =\frac{2}{3} C(1-D) \underset{\sim}{\mathbf{R}}: \underset{\sim}{\boldsymbol{\alpha}} \\
R & =Q(1-D) r \\
\tilde{Y} & =\frac{1}{2} \varepsilon^{e}: \underset{\sim}{\tilde{\Lambda}}:{\underset{\sim}{\varepsilon}}^{e}+\frac{1}{3} C \underset{\sim}{\boldsymbol{\alpha}}: \underset{\sim}{\mathbf{R}}: \underset{\sim}{\boldsymbol{\alpha}}+\frac{1}{2} Q r^{2}
\end{aligned}
$$

where tensors with the notation () refers to previously deviatoric quantities but are now mapped onto the plane stress subspace.

$\underset{\sim}{\tilde{\Lambda}}$ is a the fourth order elasticity tensor defined in the plane stress subspace by $\underset{\sim}{\tilde{\Lambda}}=2 \mu \underset{\sim}{\mathbf{1}}+\bar{\lambda} \underset{\sim}{\mathbf{1}} \otimes \underset{\sim}{\mathbf{1}}$ and $\bar{\lambda}=\frac{\nu E}{1-\nu^{2}}$ 
The yield surface and the dissipation potential are written in the general format as follows (for a Norton law),

$$
\begin{aligned}
f & =\frac{\|\boldsymbol{\sigma}-\tilde{\sim}\|-R}{\sqrt[\sim]{1-D}}-\sigma_{y} \leq 0 \\
\Phi & =\frac{K}{n+1}\left\langle\frac{f}{K}\right\rangle^{n+1}+\Phi_{D}
\end{aligned}
$$

that can be rewrite as,

$$
\begin{aligned}
\Phi= & \frac{K}{n+1}\left\langle\frac { 1 } { K } \left( f+\frac{3}{4} \frac{a}{C(1-D)} \underset{\sim}{\tilde{\mathbf{X}}}: \underset{\sim}{\mathbf{P}}: \underset{\sim}{\tilde{\mathbf{X}}}\right.\right. \\
& -\frac{1}{3} C a(1-D) \underset{\sim}{\boldsymbol{\alpha}}: \underset{\sim}{\mathbf{R}}: \underset{\sim}{\boldsymbol{\alpha}} \\
& \left.\left.+\frac{1}{2} \frac{b}{Q(1-D)} R^{2}-\frac{1}{2} b Q(1-D) r^{2}\right)\right\rangle^{n+1} \\
& +\frac{\dot{p}}{(1-D)^{\beta}}\left\langle\frac{\tilde{Y}-Y_{0}}{S}\right\rangle^{s+1} \frac{S}{s+1}
\end{aligned}
$$

where for each second order tensor $\underset{\sim}{\mathbf{T}}$, the norm $\|\underset{\sim}{\mathbf{T}}\|$ is now defined by $\|\underset{\sim}{\mathbf{T}}\|=$ $\sqrt{\frac{3}{2} \underset{\sim}{\mathbf{T}}: \underset{\sim}{\mathbf{P}}: \underset{\sim}{\mathbf{T}} \text {. }}$

The evolution equations are derived from the dissipation potential and Equations [6] to [8] become,

$$
\begin{aligned}
\stackrel{\sim}{\dot{\varepsilon}}^{v p} & =\frac{\dot{\lambda}_{v p}}{\sqrt{1-D}} \underset{\sim}{\mathbf{P}}: \underset{\sim}{\tilde{\sim}} \\
\dot{\sim} & =\dot{\lambda}_{v p}\left(\frac{\underset{\sim}{\mathbf{P}}: \underset{\sim}{\tilde{\mathbf{n}}}}{\sqrt{1-D}}-a \underset{\sim}{\boldsymbol{\alpha}}\right) \\
\dot{r} & =\dot{\lambda}_{v p}\left(\frac{1}{\sqrt{1-D}}-b r\right) \\
\dot{D} & =\frac{\dot{\lambda}_{v p}}{(1-D)^{\beta+\frac{1}{2}}}\left\langle\frac{\tilde{Y}-Y_{0}}{S}\right\rangle^{s}
\end{aligned}
$$

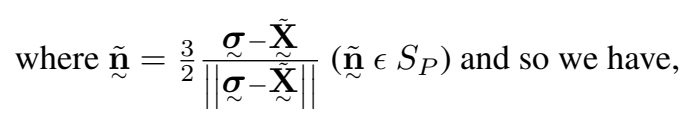




$$
\begin{aligned}
\dot{p} & =\sqrt{\frac{2}{3} \dot{\boldsymbol{\varepsilon}}^{v p}: \dot{\sim}^{v p}} \\
& =\sqrt{\frac{2}{3} \frac{\dot{\lambda}_{v p}^{2}}{1-D} \underset{\sim}{\tilde{\mathbf{n}}}: \underset{\sim}{\mathbf{P}}: \underset{\sim}{\tilde{\mathbf{n}}}} \\
& =\frac{\dot{\lambda}_{v p}}{\sqrt{1-D}}
\end{aligned}
$$

\section{- Return mapping}

Following this idea, it is now possible to realize the return mapping in the plane stress subspace applied to the present model. The asymptotic and the purely implicit backward Euler integration schemes are used to write,

$$
\begin{aligned}
& {\underset{\sim}{\boldsymbol{\alpha}+1}}_{n+1}=\boldsymbol{\sim}_{n} e^{-a \Delta \lambda_{v p}}+\frac{\underset{\sim}{\mathbf{P}}:{\underset{\sim}{\sim}}_{n+1}}{a \sqrt{1-D_{n+1}}}\left(1-e^{-a \Delta \lambda_{v p}}\right) \\
& r_{n+1}=r_{n} e^{-b \Delta \lambda_{v p}}+\frac{1}{b \sqrt{1-D_{n+1}}}\left(1-e^{-b \Delta \lambda_{v p}}\right) \\
& \Delta{\underset{\sim}{\tilde{\varepsilon}}}^{v p}=\Delta \lambda_{v p} \frac{\underset{\sim}{\mathbf{P}}:{\underset{\sim}{\tilde{\mathbf{n}}}}_{n+1}}{\sqrt{1-D_{n+1}}} \\
& D_{n+1}=D_{n}+\frac{\Delta \lambda_{v p}}{\left(1-D_{n+1}\right)^{\beta+\frac{1}{2}}}\left\langle\frac{\tilde{Y}_{n+1}\left(D_{n+1}, \Delta \lambda_{v p}\right)-Y_{0}}{S}\right\rangle^{s}
\end{aligned}
$$

Following the same procedure, one can use the elastic prediction and plastic correction method with closest point projection algorithm. However, the main difference between algorithms CP2 and CP3 is the number of unknowns. In this formulation, the only one unknown is $\Delta \lambda_{v p}$ and we realize the classical elastic prediction as in algorithm CP1 (Section 2.3.1).

$$
\boldsymbol{\sigma}_{n+1}^{\star}=\left(1-D_{n}\right)\left(2 \mu \varepsilon_{n+1}^{\star}-\lambda \operatorname{Tr}{\underset{\sim}{\varepsilon_{n+1}}}_{\sim}^{\star}\right)
$$


Consequently, stress tensor at time $t_{n+1}$ can be linked with its prediction,

$$
{\underset{\sim}{\boldsymbol{\sigma}_{n+1}}}=\left(1-D_{n+1}\right)\left(\frac{\underset{\sim}{\boldsymbol{\sigma}}}{1-D_{n}}-2 \mu \frac{\Delta \lambda_{v p}}{\sqrt{1-D_{n+1}}} \underset{\sim}{\mathbf{P}}: \underset{\sim n+1}{\tilde{\mathbf{n}}_{n+1}}\right)
$$

with the notation ${\underset{\sim}{\mathbf{Z}}}_{n+1}={\underset{\sim}{n+1}}_{\boldsymbol{\sim}_{n+1}}$, coming from Equations $[48]$ and $[53]$, we have :

$$
\begin{aligned}
& {\underset{\sim}{\tilde{\mathbf{Z}}}}_{n+1}=\left(1-D_{n+1}\right)\left(\frac{\underset{\sim}{\boldsymbol{\sigma}}{ }_{n+1}^{\star}}{1-D_{n}}-2 \mu \frac{\Delta \lambda_{v p}}{\sqrt{1-D_{n+1}}} \underset{\sim}{\mathbf{P}}: \underset{\sim}{\tilde{\mathbf{n}}_{n+1}}\right) \\
& -\frac{2}{3} C\left(1-D_{n+1}\right)\left(\underset{\sim}{\mathbf{R}}: \boldsymbol{\alpha}_{n} e^{-a \Delta \lambda_{v p}}\right. \\
& \left.+\frac{\tilde{\mathbf{n}}_{n+1}}{a \sqrt{1-D_{n+1}}}\left(1-e^{-a \Delta \lambda_{v p}}\right)\right)
\end{aligned}
$$

The Equation [54] is solely dependent on the viscoplastic multiplier which is determined by enforcing the condition $\bar{f}_{v}=0$ (Equation [55]),

$$
\begin{gathered}
\bar{f}_{v}=\frac{\left\|\tilde{\mathbf{z}}_{n+1}\right\|}{\sqrt{1-D_{n+1}}}-\sqrt{1-D_{n+1}} Q r_{n} e^{-b \Delta \lambda_{v p}} \\
-\frac{Q}{b}\left(1-e^{-b \Delta \lambda_{v p}}\right)-\sigma_{y}-\sigma_{v_{n+1}}=0
\end{gathered}
$$

With the same assumption for the damage evolution equation (Equation [27]), the only one non linear scalar equation that has to be solved is Equation [55]. Algorithm 2 summarizes this algorithm.

\section{Numerical validation}

To validate the different formulations in plane stress, first we show the influence of finite strains on the algorithms stability. Then, we compare the mechanical response of an entirely meshed tension sample in 3D and 2D to point out the accuracy of plane stress formulation. 
$\overline{\text { Algorithm } 2 \text { Closest point projection algorithm in plane stress with projection-based }}$ formulation (CP3)

$$
\begin{aligned}
& \text { 1. Initialize } \Delta \lambda_{v p}=0, \underset{D_{n+1}^{v p}}{\boldsymbol{\varepsilon}_{n}}={\underset{\sim}{v}}_{n}^{v p},{\underset{\sim}{\tilde{\mathbf{X}}}}_{n+1}=\tilde{\sim}_{n}, \\
& R_{n+1}=R_{n}, D_{n+1}=D_{n}
\end{aligned}
$$

2. Compute the trial state

3. Check yield condition equation

$f_{n+1}^{\star}=\frac{\left\|{\underset{\sim}{\boldsymbol{\sigma}_{n+1}^{\star}}}_{n}-{\underset{\sim}{\mathbf{X}}}_{n}\right\|}{\sqrt{1-D_{n}}}-\sigma_{y}$

4. IF $f_{n+1}^{\star} \leq T O L$ THEN

Set $(.)_{n+1}=(.)_{n+1}^{\star}$

\section{ELSE}

5. Iterate WHILE $f_{v}^{(i)}\left(\Delta \lambda_{v p}^{(i)} ; D_{n+1},{\underset{\sim}{\mathbf{Z}}}_{n+1}\right) \geq$ TOL

Compute ${\underset{\sim}{\mathbf{n}}}_{n+1}$ and $D_{n+1}$

Calculate the gradient of the flow rule and hardening laws

$$
\begin{aligned}
& \delta \Delta \lambda_{v p}=-\bar{f}_{v}^{(i)}\left(\frac{\partial \bar{f}_{v}^{(i)}}{\partial \Delta \lambda_{v p}}\right)^{-1} \\
& \Delta \lambda_{v p}^{(i+1)}=\Delta \lambda_{v p}^{(i)}+\delta \Delta \lambda_{v p}
\end{aligned}
$$

6. Update state variables and normal

7.Set $\mathrm{i}=\mathrm{i}+1$ and GOTO 5.

ENDIF

\subsection{Influence of finite strains}

Only one element is loaded in the direction 1 (Figure 1) with four different strain rates lying from $\dot{\varepsilon}=10^{-1} s^{-1}$ to $\dot{\varepsilon}=10^{-4} s^{-1}$ with and without finite strains. As mentioned in Section 2.3.1, we can see that the algorithm CP1 with finite strains, as expected, leads to an unstable response clearly visible as the strain rates increase (Figure 2). This numerical instability disappear completely under the small strain assumption as shown in 3 


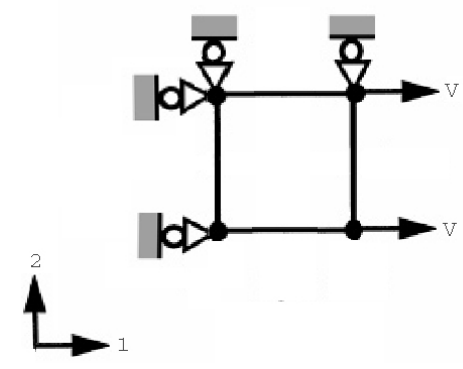

Figure 1. Uniaxial tension of an RVE
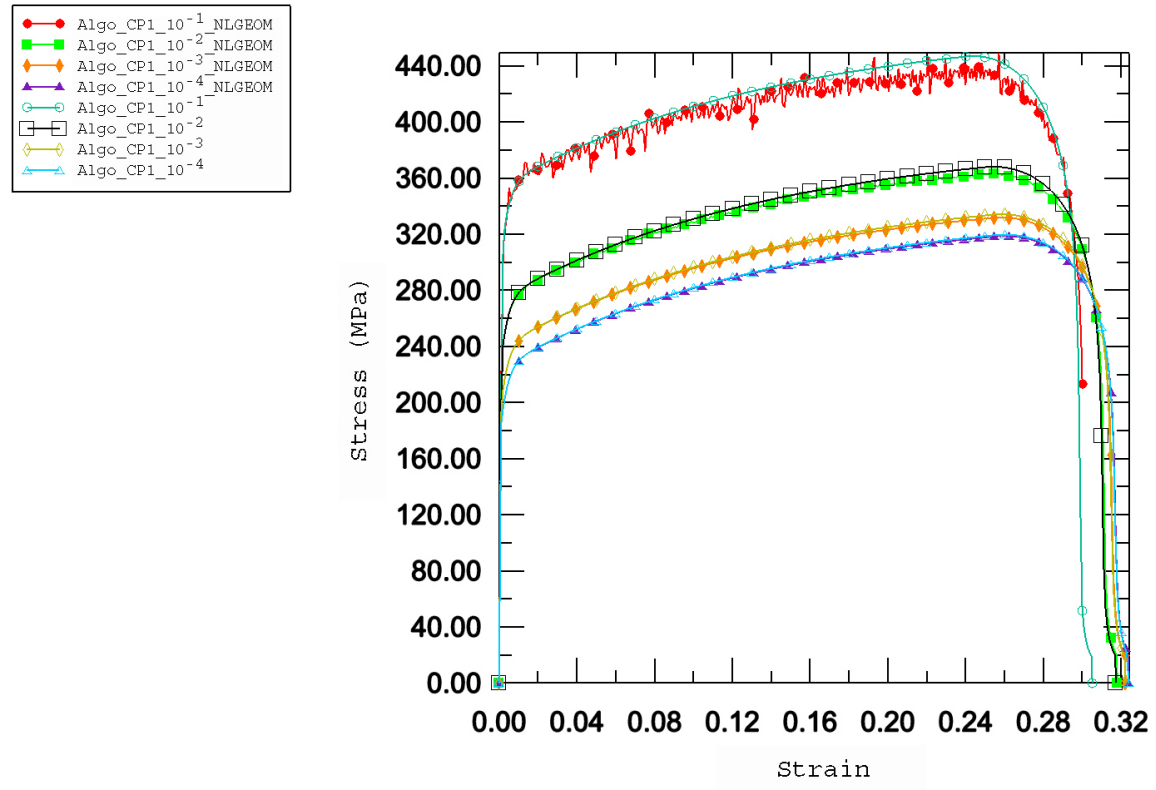

Figure 2. Comparison of the mechanical response of algorithm CP1, uniaxial tension for different $\dot{\varepsilon}\left(10^{-4}, 10^{-3}, 10^{-2}, 10^{-1} \mathrm{~s}^{-1}\right)$ with and without finite strains (non-linear geometry activated (NLGEOM))

\subsection{Comparison between the three algorithms}

With the same procedure, we can now compare the three algorithms responses to the uniaxial loading with (Figure 4) and without (Figure 3) finite strains.

In small strains, the mechanical response of the three algorithms are exactly the same with a very small difference in the localization stage between the first algorithm 

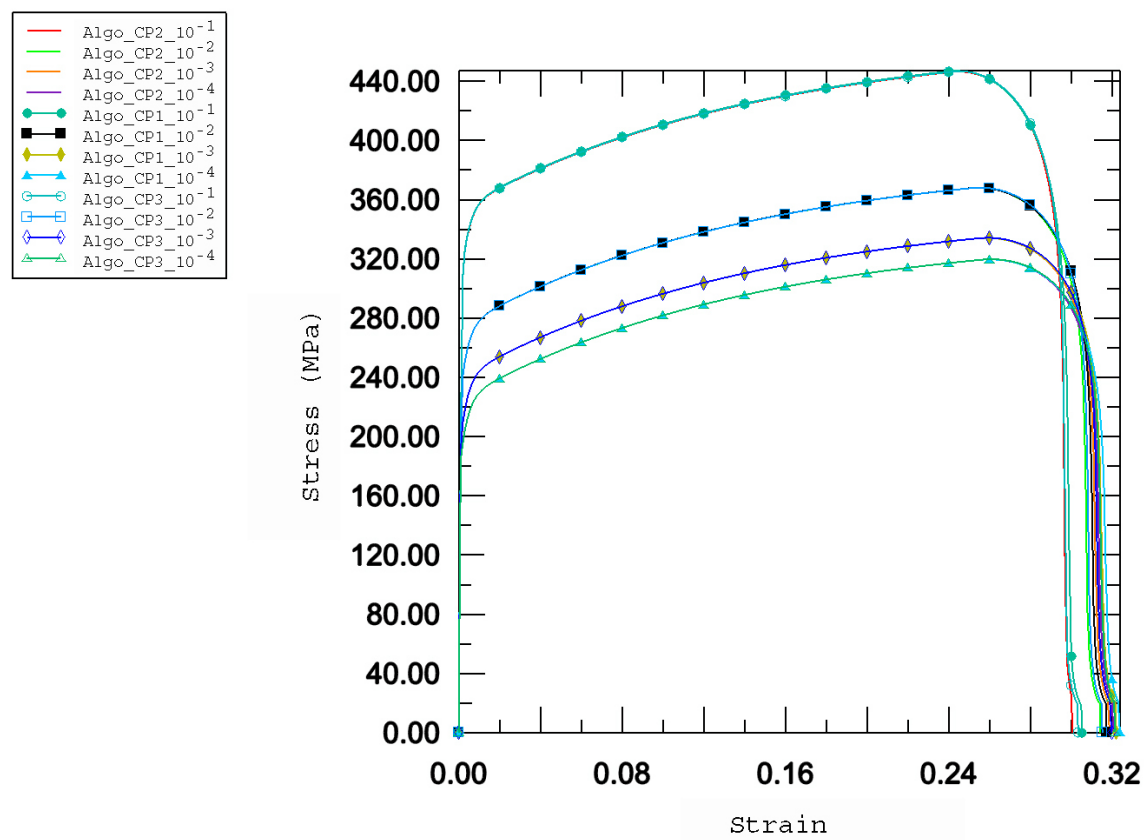

Figure 3. Comparison of the mechanical response in uniaxial tension for algorithms $C P 1, C P 2$ and $C P 3$, and different $\dot{\varepsilon}\left(10^{-4}, 10^{-3}, 10^{-2}, 10^{-1} s^{-1}\right)$ without finite strains (non-linear geometry activated (NLGEOM))

and the two others. In finite strains, the first unstable algorithm response is seen to fall around the two stable others for the strain rates $\dot{\varepsilon}=10^{-1} \mathrm{~s}^{-1}$ and $10^{-2} \mathrm{~s}^{-1}$. For $\dot{\varepsilon}=10^{-3} s^{-1}$ and $10^{-4} s^{-1}$, the loading step is small and leads to a response that seems to be stable.

\subsection{Comparison with the $3 D$ case}

To compare the force-displacement responses of the above algorithms in plane stress, we compare them with the simulation of the 3D case. The geometry used for that simulation is described in Figure 5 for the 3D case and in Figure 6 for the plane stress case.

In Figure 7, we compare the force-displacement curves up to failure with the three algorithms in $3 \mathrm{D}$ and plane stress. To compare structural response of the three algorithms, we have chosen to use the lowest strain rate loading (velocity $=10^{-4} \mathrm{~mm} . \mathrm{s}^{-1}$ ) to have the stablest response for the first algorithm. The same routine is used for 3D and plane stress cases. As expected, the mechanical response is overestimated in 

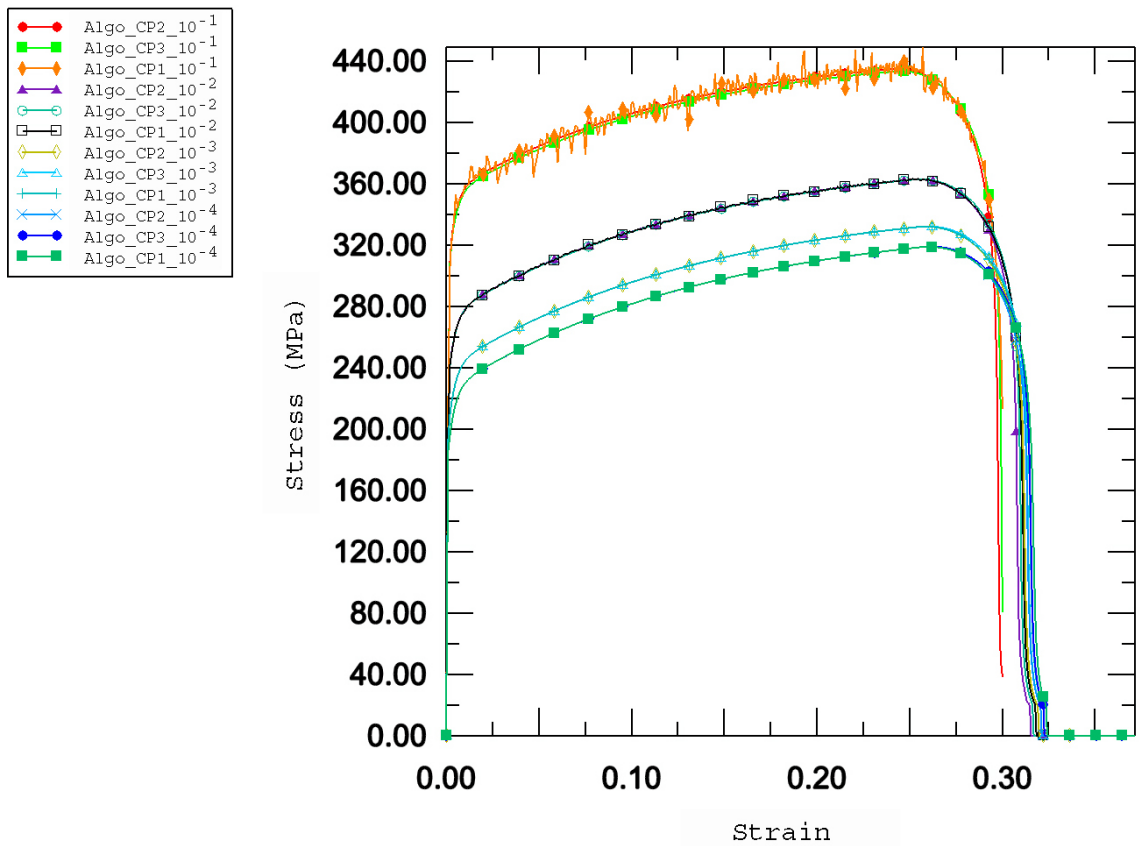

Figure 4. Comparison of the mechanical response in uniaxial tension for algorithms $C P 1, C P 2$ and $C P 3$, and different $\dot{\varepsilon}\left(10^{-4}, 10^{-3}, 10^{-2}, 10^{-1} \mathrm{~s}^{-1}\right)$ with finite strains (non-linear geometry activated (NLGEOM))

plane stress for the first algorithm while the two other ones are really close to the 3D response. Consequently, the algorithm $\mathrm{CP} 1$ isn't just unstable but also wrong for a structural simulation with element rotation. The algorithms CP2 and CP3 exhibit a small difference at the very end of the simulation. This may be caused by mesh dependency in the softening stage for this fully local formulation. The main advantage of the plane stress simulation is obviously the computational cost efficiency as can be seen in Table 1. Algorithm CP1 isn't really efficient compared to the two others. The computational cost of algorithms CP2 and CP3 is close and the gain is about $29 \%$ between 3D and 2D simulations.

\subsection{Deep drawing test}

To show the model accuracy in both 2D and 3D cases for the algorithm $\mathrm{CP} 3$, the deep drawing test of cylindrical cup, presented in Figure 8 , is worked out.

For the same test, 2D and 3D force-displacement responses are very close (Figure 97. The 2D simulation overestimates the maximum loading force of about $4 \%$ but is 


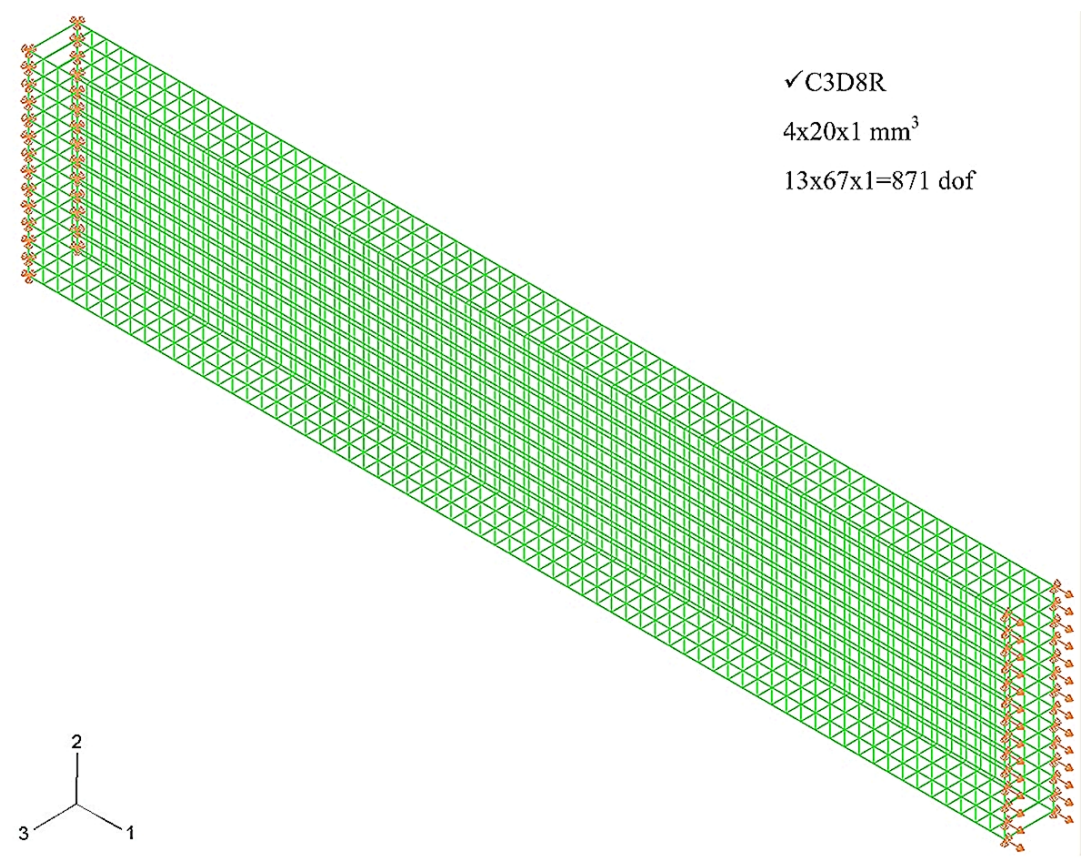

Figure 5. Uniaxial tensile test meshed with $3 D$ elements C3D8R and 871 dof

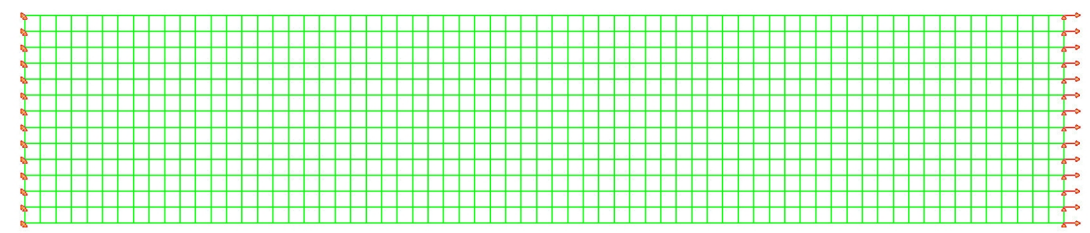

$\checkmark \mathrm{CPS} 4 \mathrm{R}$

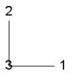

$4 \times 20 \mathrm{~mm}^{2}$

$13 \times 67=871$ dof

Figure 6. Uniaxial tensile test meshed with $2 D$ elements CPS4R and 871 dof 


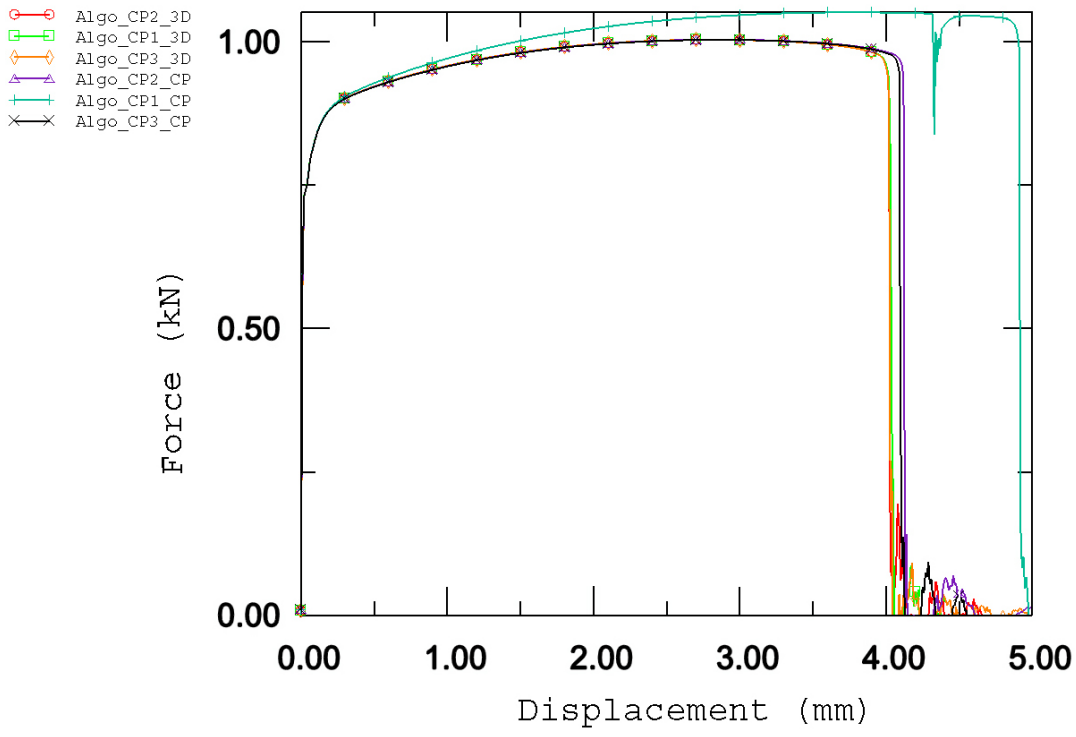

Figure 7. Force displacement for velocity $=10^{-4} \mathrm{~mm} \cdot \mathrm{s}^{-1}$ in $3 \mathrm{D}$ and plane stress cases with the three algorithms in finite strain

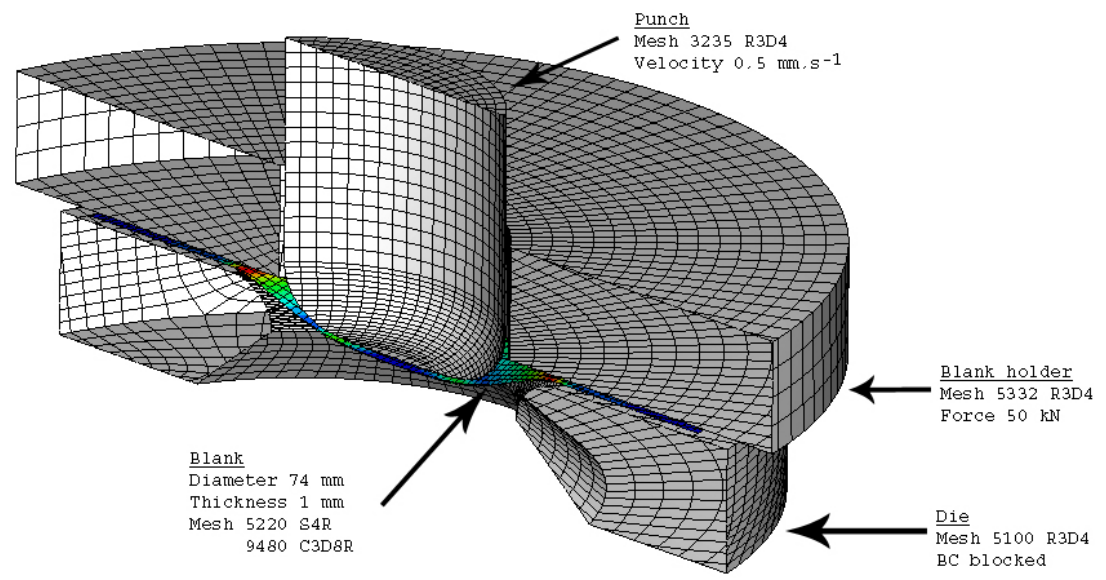

Figure 8. Schematic representation of deep drawing test with boundary conditions 


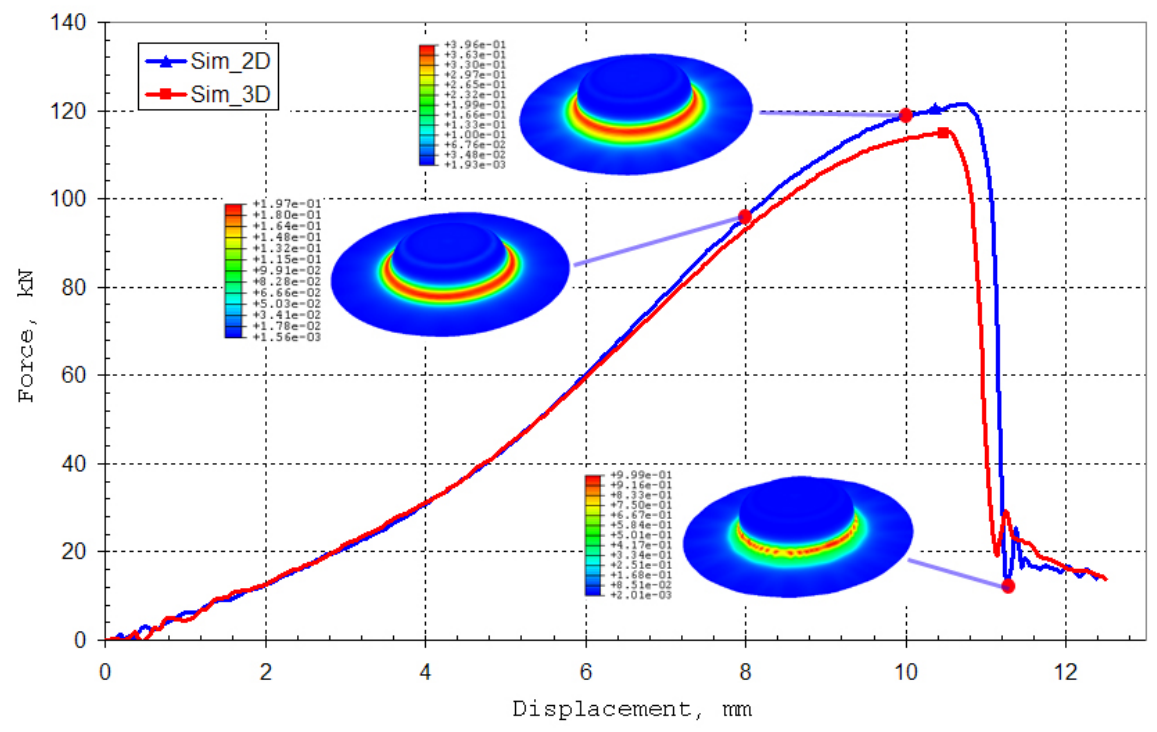

Figure 9. Comparison between force-displacement responses of $2 D$ and $3 D$ cases (algorithm CP3) for the deep drawing test and damage distribution for three steps of the $2 D$ simulation
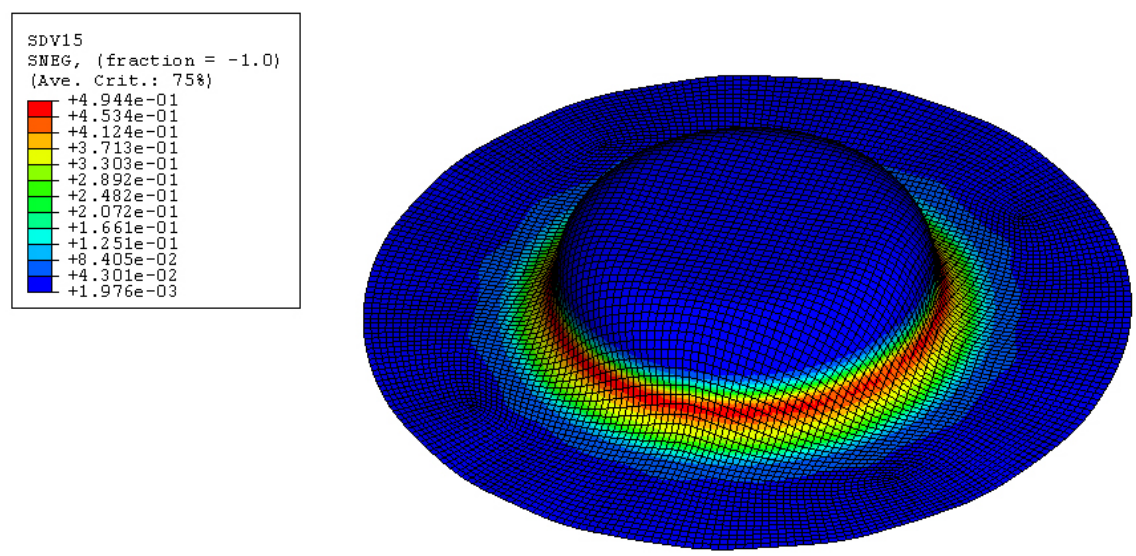

Figure 10. Damage distribution for a $10 \mathrm{~mm}$ displacement of the punch in plane stress (back side) 
362 European Journal of Computational Mechanics. Volume 20 - No. 5-6/2011

Table 1. Comparison of computational costs of the three algorithms for uniaxial tensile test in finite strain for a three millimeter displacement

\begin{tabular}{llll}
\hline & CP1 & CP2 & CP3 \\
\hline 3D & $00: 07: 52$ & $00: 07: 17$ & $00: 07: 28$ \\
plane stress & $00: 07: 21$ & $00: 05: 08$ & $00: 05: 17$ \\
CPU Time saved & $10 \%$ & $29 \%$ & $29 \%$ \\
\hline
\end{tabular}
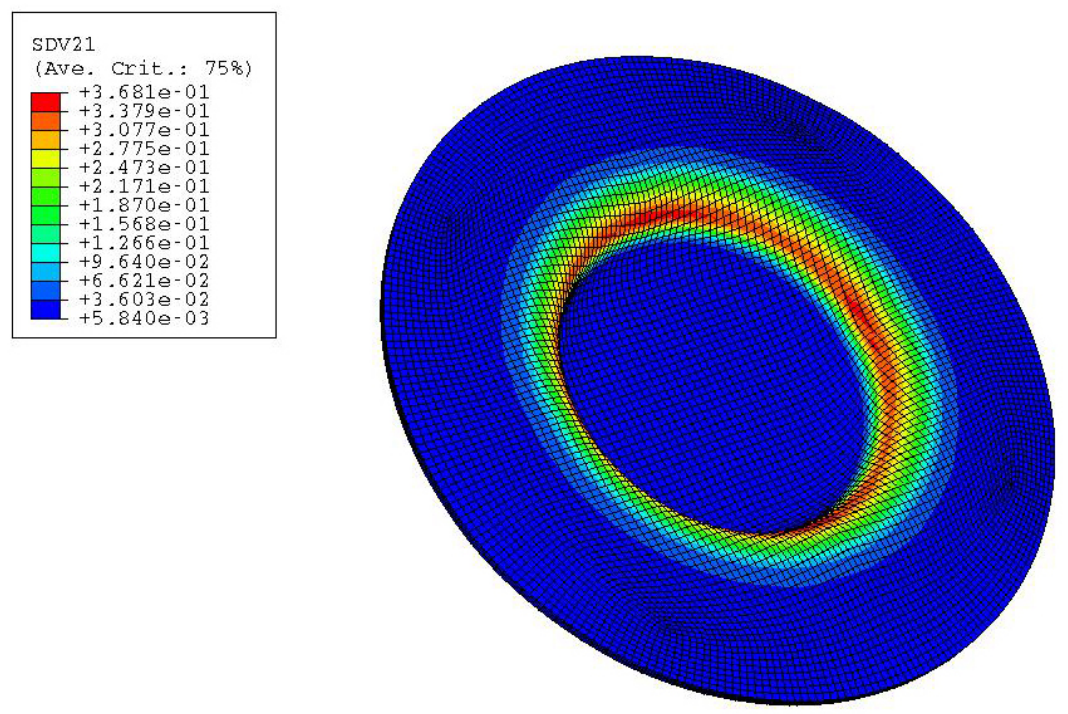

Figure 11. Damage distribution for a $10 \mathrm{~mm}$ displacement of the punch in $3 D$ (back side)

very efficient to save CPU time (03:10:02 for the 3D simulation versus 00:40:32 for the $2 \mathrm{D}$ one). This gap starts from a displacement of $6 \mathrm{~mm}$ and continue to grow up to failure. However, the total failure of the blank is well described in time and space, since the failure time and location are almost the same between 2D and 3D (Figure 10 and 11. 


\section{Conclusion}

To conclude, an elasto-viscoplastic model with non linear hardenings, fully coupled with damage is formulated. The plane stress assumption, needed for shell structures, is treated using three different algorithms and compared together with the $3 \mathrm{D}$ case. The first one, CP1, only imposes an additional equation coming from $\sigma_{33}=0$ and is shown to be unstable with finite strain. The second, CP2, uses elastic prediction directly on two variables in plane stress $\left(\Delta \lambda_{v p}\right.$ and $\left.\epsilon_{33}\right)$ and explicitly shows the importance of plane stress constraint on the plastic flow direction (rotation of the normal during the plastic correction). The third one, CP3, is a projection-based algorithm, following the original idea of Simo, and have the main advantage of leading to only one scalar equation to solve with the closest point projection scheme. This last one has a good accuracy and stability but is a little more CPU time consuming. Numerical simulations show that the two last algorithms are in good agreement, in the plane stress case, with a 3D thin plate response. Moreover, the projection based algorithm is very interesting because it can easily address other problems, not discussed here, like quadratic anisotropic yield criterion with or without plane stress condition by only changing the projection matrix (Paris, 2008).

To conclude, we have seen that the use of shell elements to describe structural test such as deep drawing test of cylindrical cup, can be efficiently used to have a fast local and global response up to failure. However, some specific metal forming conditions or tests as well as damage parameters could influence the stress state when damage occurs. In that specific conditions, the out of plane stress can become non negligible and the $3 \mathrm{D}$ formulation must be preferred.

\section{References}

Aravas N., " On the numerical integration of a class of pressure dependent plasticity models,', Int. J. Numer. Meth. Engng., vol. 24, p. 1395-1416, 1987.

Aravas N., Aifantis E., "On the Geometry of Slip and Spin in Finite Plastic Deformation”, Int. J. of Plasticity, vol. 7, p. 141-160, 1991.

Badreddine H., Elastoplasticité anisotrope endommageable en grandes déformations : aspects théoriques, numériques et applications, $\mathrm{PhD}$ thesis, Université de Technologie de Troyes, École Nationale d'Ingénieurs de Monastir, 2006.

Belytschko T., Liu W., Moran B., Nonlinear finite elements for continua and structures, John Wiley, New York, 2000.

Besson J., Foerch R., “Large scale object-oriented finite element code design', Comput. Methods Appl. Mech. Engrg., vol. 142, p. 165-187, 1997.

Chaboche J., "A review of some plasticity and viscoplasticity constitutive theories', Int. J. of Plasticity, 2007.

Crisfield M., Non-linear Finite Element Analysis of Solids and Structures, vol. 1-2, John Wiley, New York, 1991. 
364 European Journal of Computational Mechanics. Volume 20 - No. 5-6/2011

De Borst R., " The zero-normal-stress condition in plane stress and shell elastoplasticity", Comm. Appl. Num. Meth, vol. 7, p. 29-33, 1991.

Dodds R. H., “ Numerical techniques for plasticity computations in finite element analysis', Computers and Structures, vol. 26 n 5, p. 767-779, 1987.

Doghri I., Mechanics of Deformable Solids, Springer-Verlag, Berlin, 2000.

Doghri I., Billardon R., "Investigation of localization due to damage in elasto-plastic materials', Mechanics of Materials, vol. 19, p. 129-149, 1995.

Dogui A., Plasticité anisotrope en grandes déformations, $\mathrm{PhD}$ thesis, Université Claude Bernard, Lyon, 1989.

Fuschi P., Peric D., Owen D., "Studies on generalized mid point integration in rate-independent plasticity with reference to a plane stress J2-flow theory|', Computers and Structures, vol. 43, p. 1117-1133, 1992.

Hammi Y., Simulation numérique de l'endommagement des procédés de mise en forme, $\mathrm{PhD}$ thesis, Université de Technologie de Compiègne, 2000.

Ibrahimbegovic A., Mécanique non linéaire des solides déformables : formulation théorique et résolution numérique par éléments finis, Hermès science, 2006.

Kumar P., Nukala V., “A return mapping algorithm for cyclic viscoplastic constitutive models,' Comput. Methods Appl. Mech. Engrg., vol. 195, p. 148-178, 2005.

Lee J., Fenves G., “ A return-mapping algorithm for plastic-damage models: 3-D and plane stress formulation', Int. J. Numer. Methods Engrg, vol. 50, p. 487-506, 2001.

Lee S., Yoon M., Yang D., “A stress integration algorithm for plane stress elastoplasticity and its application to explicit finite element analysis of sheet metal forming processes', Computers and Structures, vol. 66, p. 301-311, 1998.

Lemaître J., Chaboche J., Mécanique des matériaux solides, Dunod, France, 1985.

Lestriez P., Modélisation numérique du couplage thermomécanique endommagement en transformations finies: Application à la mise en forme, $\mathrm{PhD}$ thesis, Université de Technologie de Troyes, 2003.

Lestriez P., Saanouni K., Mariage J., Cherouat A., “ Numerical prediction of damage in metal forming process including thermal effects”, Int. J. of Damage Mechanics, vol. 13, p. 59-80, 2004.

Millard A., " Numerical algorithms for plane stress elastoplasticity: review and recommendation”, Computational Plasticity 4th Int. Conf., p. 237-247, 1995.

Montans F., "Implicit plane stress algorithm for multilayer J2-plasticity using the PragerZiegler translation rule,', Int. J. Numer. Meth. Engng, vol. 59, p. 409-418, 2004.

Ortiz M., Simo J., "Analysis of a new class of integration algorithms for elastoplastic constitutive relations,', Int. J. Num. Meth. Engng., vol. 23 n³, p. 353-366, 1986.

Paris T., Modélisation du comportement mécanique des liaisons soudées hétérogènes Ta/TA6V : Comportement et critère de rupture., PhD thesis, Université de Technologie de Troyes, 2008.

Saanouni K., Chaboche J., “ Computational damage mechanics. Application to metal forming, Numerical and Computational methods", Elsevier Oxford, vol. 3, n 7, p. 321-376, 2003.

Saanouni K., Forster C., BenHatira F., “ On the inelastic flow with damage”, Int. J. Damage Mech, vol. 3, n 2, p. 140-169, 1994. 
Sawyer J., Wang C., Jones R., “ An implicit algorithm using explicit correctors for the kinematic hardening model with multiple back stresses', Int. J. Numer. Methods Engrg, vol. 50, p. 2093-2107, 2001.

Simo J., Govindjee S., “ Exact closed-form solution of the return mapping algorithm in plane stress elasto-viscoplasticity”, Eng. Comput., vol. 5, p. 254-258, 1988.

Simo J., Hughes T., Computational Inelasticity, Springer Verlag, 1997.

Simo J., Taylor R., “ A return mapping algorithm for plane stress elastoplasticity”, Int. J. Num. Meth. Engng., vol. 22, p. 649-670, 1986.

Singh n. A., Pandey P., “ An implicit integration algorithm for plane stress damage coupled elastoplasticity]', Mechanics Research Communications, vol. 26, p. 693-700, 1999.

Valoroso N., Rosati L., " Consistent derivation of the constitutive algorithm for plane stress isotropic plasticity. Part I Theoretical formulation]', International Journal of Solids and Structures, vol. 46, p. 74-91, 2009a.

Valoroso N., Rosati L., “ Consistent derivation of the constitutive algorithm for plane stress isotropic plasticity. Part II Computational issues', International Journal of Solids and Structures, vol. 46, p. 92-124, 2009b.

Walker K., Freed A., "Asymptotic Integration Algorithms for Nonhomogeneous, Nonlinear, First Order, Ordinary Differential Equations,', Engineering Science Software, NASA Technical Memorandum, 1991.

Waltz G., Hornberger K., Stamm H., “ An implicit integration algorithm for plane stress viscoplastic constitutive equations,', Computers and Structures, vol. 3, p. 539-546, 1990.

\section{Appendix : Detailed development of algorithm CP2}

As mentioned before, the best way to ensure a stable response in any case is to realize both an elastic prediction on $\Delta \lambda_{v p}(=0)$ and $\Delta \varepsilon_{33}\left(=\Delta \varepsilon_{33}^{\star}\right)$. The trial stress and strain are now written as follow:

$$
{\underset{\sim}{\boldsymbol{\sigma}^{*}}}_{n+1}^{\star}=\left(1-D_{n}\right) \underset{\sim}{\boldsymbol{\Lambda}}:{\underset{\sim}{\boldsymbol{\varepsilon}_{n+1}^{\star}}}^{\star}
$$

and

$$
{\underset{\sim}{\varepsilon_{n+1}}}_{n}^{\star}={\underset{\sim}{\boldsymbol{\varepsilon}_{n+1}}}_{e}^{e}+\Delta \varepsilon_{33}^{\star} \underset{\sim 3}{\mathbf{1}}
$$

where $\underset{\sim 3}{\mathbf{1}}$ refers to the following unit vector :

$$
{\underset{\sim}{3}}_{3}=\left(\begin{array}{l}
0 \\
0 \\
1 \\
0
\end{array}\right)
$$


The tensor $\bar{\varepsilon}_{n+1}^{e}$ is the known strain tensor (imposed load) passed to the user subroutine by the finite element software in the plane stress case. All the components of this tensor are known at time $t_{n+1}$ except $\varepsilon_{33, n}$ which is known at $t_{n}$ :

$$
\bar{\varepsilon}_{n+1}^{e}=\left(\begin{array}{c}
\varepsilon_{11, n+1}^{e} \\
\varepsilon_{22, n+1}^{e} \\
\varepsilon_{33, n}^{e} \\
\varepsilon_{12, n+1}^{e}
\end{array}\right)
$$

The elastic deformation and its deviatoric part can be written :

$$
\begin{aligned}
& {\underset{\sim}{\varepsilon_{n+1}}}_{e}^{e}={\underset{\sim}{\varepsilon_{n}}}_{e}^{e}-\Delta \underset{\sim}{\varepsilon}-\Delta{\underset{\sim}{\varepsilon}}^{v p} \\
& =\bar{\sim}_{n+1}^{e}+\Delta \varepsilon_{33} \underset{\sim 3}{\mathbf{1}_{3}}-\Delta{\underset{\sim}{\varepsilon}}^{v p} \\
& =\varepsilon_{n+1}^{\star}-\Delta \varepsilon_{\sim}^{v p}+\left(\Delta \varepsilon_{33}-\Delta \varepsilon_{33}^{\star}\right){\underset{\sim}{1}}_{3}
\end{aligned}
$$

and

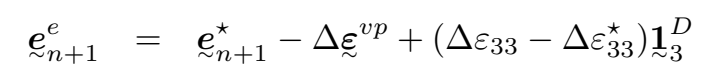

From Equation [61], we can write the spheric and deviatoric part of the stress tensor :

$$
\begin{aligned}
{\underset{\sim}{n+1}}_{n}= & \left(1-D_{n+1}\right)\left[\frac{\mathbf{\sim}_{n+1}^{\star}}{1-D_{n}}-2 \mu \Delta{\underset{\sim}{\varepsilon}}^{v p}+K \operatorname{Tr}\left({\underset{\sim}{n+1}}_{n+1}^{\star}\right) \underset{\sim}{\mathbf{1}}\right. \\
& \left.+2 \mu\left(\Delta \varepsilon_{33}-\Delta \varepsilon_{33}^{\star}\right) \underset{\sim}{\mathbf{1}} D+\left(\Delta \varepsilon_{33}-\Delta \varepsilon_{33}^{\star}\right) \underset{\sim}{\mathbf{1}}\right]
\end{aligned}
$$

where $\mathrm{K}$ is the bulk modulus and $\underset{\sim 3}{\underset{1}{D}}$ refers to the deviatoric part of $\underset{\sim 3}{\underset{1}{1}}$.

$$
\begin{aligned}
& {\underset{\sim}{\mathbf{S}_{n+1}}}=\left(1-D_{n+1}\right)\left[\frac{\underset{\sim}{\mathbf{S}_{n+1}^{\star}}}{1-D_{n}}-2 \mu \Delta{\underset{\sim}{v i p}}^{v p}+2 \mu\left(\Delta \varepsilon_{33}-\Delta \varepsilon_{33}^{\star}\right)_{\sim}^{D}\right] \\
& \sigma_{n+1}^{D}=\left(1-D_{n+1}\right)\left[\frac{\sigma_{n+1}^{H \star}}{1-D_{n}}+3 K\left(\Delta \varepsilon_{33}-\Delta \varepsilon_{33}^{\star}\right)\right]
\end{aligned}
$$


with $\sigma_{n+1}^{H}=\operatorname{Tr}\left(\boldsymbol{\sigma}_{n+1}\right)$.

In order to express the normal to the yield surface, we use the notation $\mathbf{Z}_{n+1}=$ $\mathbf{S}_{n+1}-\mathbf{X}_{n+1}$ and write,

$$
\begin{aligned}
{\underset{\sim}{\mathbf{Z}}}_{n+1}= & \left(1-D_{n+1}\right) \frac{\underset{\mathbf{S}_{n+1}^{\star}}{\star}-\mathbf{\sim}_{n} e^{-a \Delta \lambda_{v p}}}{1-D_{n}} \\
& -2 \sqrt{1-D_{n+1}}\left[\mu \Delta \lambda_{v p}+\frac{C\left(1-e^{-a \Delta \lambda_{v p}}\right)}{3 a}\right]{\underset{\sim}{n}}_{n+1} \\
& +2 \mu\left(1-D_{n+1}\right)\left(\Delta \varepsilon_{33}-\Delta \varepsilon_{33}^{\star}\right){\underset{\sim}{1}}^{D}
\end{aligned}
$$

Noting that ${\underset{\sim}{n+1}}_{n+1}=\frac{3}{2} \frac{\widetilde{\mathbf{Z}}_{n+1}}{\|\underset{\sim}{\mathbf{Z}} n+1\|}$, we obtain the following tensorial equation,

$$
\begin{aligned}
\underset{\sim}{\mathbf{n}_{n+1}} & \quad\left[\frac{2}{3}\left\|{\underset{\sim}{\mathbf{Z}}}_{n+1}\right\|+2 \sqrt{1-D_{n+1}}\left(\mu \Delta \lambda_{v p}+\frac{C\left(1-e^{-a \lambda_{v p}}\right)}{3 a}\right)\right] \\
= & \left(1-D_{n+1}\right) \frac{{\underset{\sim}{n+1}}_{n+\underset{\sim}{\mathbf{X}} e^{-a \Delta \lambda_{v p}}}}{1-D_{n}} \\
+ & 2 \mu\left(1-D_{n+1}\right)\left(\Delta \varepsilon_{33}-\Delta \varepsilon_{33}^{\star}\right){\underset{\sim 3}{\mathbf{1}}}^{D}
\end{aligned}
$$

By taking the expression of the norm $\left\|\mathbf{Z}_{n+1}\right\|$ from the yield condition and using it in Equation [66], one can obtain,

$$
\begin{aligned}
& \mathbf{n}_{n+1} \quad\left[R_{n} e^{-b \Delta \lambda_{v p}}+\frac{1-D_{n}}{\sqrt{1-D_{n+1}}}\left(\frac{Q}{b}\left(1-e^{-b \Delta \lambda_{v p}}\right)\right.\right. \\
& \left.\left.+\frac{C}{a}\left(1-e^{-a \Delta \lambda_{v p}}\right)+\sigma_{y}+\sigma_{v_{n+1}}+3 \mu \Delta \lambda_{v p}\right)\right]= \\
& \frac{3}{2} \quad\left\|\mathbf{S}_{n+1}^{\star}-\mathbf{\sim}_{n} e^{-a \Delta \lambda_{v p}}+2 \mu\left(1-D_{n}\right)\left(\Delta \varepsilon_{33}-\Delta \varepsilon_{33}^{\star}\right) \underset{\sim 3}{\mathbf{1}_{3}^{D}}\right\|
\end{aligned}
$$

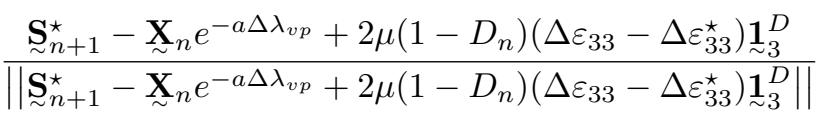

which leads to the Equations [30] and [31] 
The additional equation in the plane stress case comes from the constraint,

$$
\begin{aligned}
& \sigma_{33}=0 \\
& S_{33}+\frac{1}{3} \operatorname{Tr}{\underset{\sim}{\boldsymbol{\sigma}}}_{n+1}=0 \\
& \frac{1}{1-D_{n}}\left[\left({\underset{\sim}{n+1}}_{n+1}^{\star}\right)_{33}+\frac{1}{3} \underset{\sim n+1}{\boldsymbol{\sigma}_{n+1}}\right]-\frac{2 \mu \Delta \lambda_{v p}}{\sqrt{1-D_{n+1}}}\left({\underset{\sim}{n+1}}_{n 3}\right)_{33} \\
& \left(K+\frac{4}{3} \mu\right)\left(\Delta \varepsilon_{33}-\Delta \varepsilon_{33}^{\star}\right)=0
\end{aligned}
$$

This gives the Equation [32]

Received: 23 September 2010 Accepted: 11 July 2011 